\title{
Impacto de Covey, Riso y Sanborn en habilidades gerenciales
}

\author{
Héctor Serrano Mantilla / Jesennia Cárdenas Cobo / \\ Mariuxi Vinueza Morales / Rodolfo Robles Salguero
}

\section{RESUMEN}

El objetivo del presente estudio, consiste en demostrar precisamente la validez de la hipótesis de que existe un estrecho vínculo entre la lectura y la autonomía del estudiante de ciencias administrativas para su aprendizaje, generando y fortaleciendo sus habilidades gerenciales, llegando a predecir que el conocimiento por estos, de lecturas automotivadoras como "Aprendiendo a Quererse a sí mismo" de Walter Riso, "El factor Fred” de Mark Sanborn y "Los Siete Hábitos de la Gente altamente Efectiva" de Stephen Covey, ya sea en el colegio o en la universidad, permiten reafirmar dicha hipótesis; la metodología aplicada fue no experimental, exploratoria y descriptiva, el presente estudio se realizó en la Universidad Estatal de Milagro UNEMI, concluyendo que la lectura de textos motivacionales fortalecen sus habilidades blandas o humanas, porque aportan en su motivación y proyección como el líder que supera la zona de confort y que demuestra apertura al cambio.

PALABRAS CLAVE:

Motivación, autoestima, autoconcepto, lectura, habilidades gerenciales.

\section{ABSTRACT}

It has been hypothesized that there is a link between reading and students' autonomous learning of management science. Reading is supposed to generate and strengthen management skills. The purpose of this paper is to demonstrate the truth of this hypothesis. It has been predicted that at reading self-improvement works like The Seven Habits of Highly Effective People by Stephen Covey, Learning to Love Oneself by Walter Riso and The Fred Factor by Mark Sanborn, whether in high school or in college will lead to reaffirmation of this hypothesis. The methodology used was exploratory and descriptive, not experimental. The present study was carried out in the Milagro State University (UNEMI). The conclusion that was reached was that the reading of self-improvement texts strengthens soft or human skills because it increases motivation and leadership projection above and beyond the comfort zone and shows openness to change.

\section{KEYW/ORDS:}

Motivation, self-esteem, self-concept, reading, management skills 


\section{Introducción}

La principal problemática de investigación reside en la baja autoestima y autoeficacia detectada en los estudiantes de la Carrera de Administración de Empresas de la Universidad Estatal de Milagro (UNEMI), lo cual ha estado relacionada con el poco hábito de lectura que tienen y la escasa motivación por esta, principalmente por no haber conocido antes, lecturas auto motivadoras como son: las obras de los autores Covey, Riso y Sanborn.

El objetivo del presente trabajo consiste en demostrar precisamente la validez de la hipótesis de que existe un estrecho vínculo entre la lectura y la autonomía del estudiante para su aprendizaje, generando y fortaleciendo en sus habilidades gerenciales, llegando a predecir que el conocimiento por estas lecturas automotivadoras, como "Aprendiendo a Quererse a sí mismo" de Walter Riso, "El factor Fred" de Mark Sanborn y "Los Siete Hábitos de la Gente altamente Efectiva" de Stephen Covey, ya sea en el colegio o en la universidad, permiten reafirmar dicha hipótesis. Cuando una persona asimila estos textos está aportando a su desarrollo profesional, Frank Ponti menciona que "Tener conciencia de las capacidades personales es especialmente interesante porque permite orientar la creatividad" (Ponti, 2008, 27).

La investigación se sustenta en un marco teórico relacionado a las obras de los autores arriba mencionados; así como también de obras relacionadas con las inteligencias múltiples de Goleman, sobre liderazgo en la administración escritos por Drucker y Sutton, pensamiento lateral asimilado por De Bono y Coaching de diferentes autores.

La investigación se la realizó en la Carrera de Administración de Empresas de la UNEMI y se utilizaron varias técnicas de recolección de információn, como el cuestionario a los estudiantes de tercer a sépti- mo nivel de la carrera, la entrevista a egresados y la observación participante de los autores, para recabar los datos que permiten inferir determinados nexos entre los fenómenos investigados: lectura motivacional, autoaprendizaje y eficacia en los estudios universitarios.

En las aulas universitarias se debe lograr que los futuros administradores descubran sus fortalezas o competencias ocultas, lo cual no solo implica la lectura de un texto, sino contar con profesores coach que generen clases sinérgicas por el deseo de mejorar a sus alumnos con procesos pragmáticos, al respecto Laura Fierro Evans, identifica lo que ella llama como una "zona potencial" y para lograr esta " se ponen en marcha varios motores: La necesidad, la curiosidad, la voluntad, el deseo de logro, la asunción de riesgos y la determinación" (Fierro, 2015, 44).

Preparar a los futuros administradores a un mercado de talentos humanos muy complejo; es vital en donde ellos deberán contar con habilidades gerenciales que genere la autoestima necesaria en las personas que administran o que lideren, John $\mathrm{H}$. Zenger y Kathleen Stinnett comentan "Las personas más eficientes poseen un alto nivel de autoestima y confianza en sí mismos" (Zenger, 2013, 16).

Las habilidades técnicas son de suprema importancia en el crecimiento profesional de un administrador, deben tener un acompañamiento de las habilidades blandas o humanas si desea generar resultados excepcionales, aportando a las habilidades conceptuales inclusive, porque para lograr nuevos y más complejos objetivos requerirá el apoyo de otras personas, J. Keith Murnighan expresa lo siguiente "Cuando usted asciende ya no puede confiar en sus destrezas técnicas, usted tiene que dirigir, o mejor dicho, tiene que liderar" (Murnighan 2013,24) y Jennifer B. Kahnweiler menciona "Para motivar a las personas y obtener 
resultados se necesita algo más que experiencia técnica o dominio de la materia" (Kahnweiler, 2010, 35).

\section{Problema de investigación}

Como profesores universitarios se cuenta con un sinnúmero de experiencias producto de la interacción que se tiene con los estudiantes, con satisfacción se aprecia y valora en alto grado las fortalezas que presentan los nuevos integrantes de la universidad en la Unidad Académica de Ciencias Administrativas en la sección nocturna de la Universidad Estatal de Milagro UNEMI y de manera especial, los que han tomado la decisión de iniciar su carrera en Administración de Empresas.

De manera similar se observa debilidades en este grupo de estudiantes, si bien es cierto estas debilidades no son un elemento común pero la existencia de ellas afecta directamente a su desarrollo personal y profesional, como lo menciona Daniel Goleman en su libro La Práctica de la Inteligencia Emocional: "Hoy en día se hace cada vez más necesaria la importancia de habilidades que impliquen el control propio de las emociones" (Covey, 2004, 24), en este caso refiriéndose a lo que identifica como habilidades "Blandas" o relacionadas con las habilidades "Humanas y Conceptuales" que sinérgicamente se suman a las habilidades Técnicas (Robbins, 2009, 8), que tienen un alto impacto en la gestión de los administradores en las empresas.

En las Universidades los profesores se deben enfrentar a poderosos rivales ocultos que se encuentran en determinados alumnos, estos rivales son los paradigmas errados, arraigados y que se constituyen en barreras que parecerían impenetrables, que se los identifica como resistencias al cambio, Lidia Mauradep menciona "No hemos sido entrenados para saber qué hacer cuando los paradigmas a los que estamos habituados comienzan a quebrarse, por eso tendemos a aferrarnos a lo viejo aunque ya no sirva" (Muradep, 2015, 17).

Los docentes universitarios presencian de manera continua como determinados estudiantes no aprovechan sus fortalezas, que teniendo un potencial en su talento humano, no lo explotan porque no conocen de su existencia, o la minimizan o desvaloran ya que sus niveles de autoestima no son los mejores, requiriendo un proceso de cambio, esto permite identificar el siguiente problema:

"Determinados estudiantes que inician su proceso universitario en la Unidad Académica de Ciencias Administrativas (Administración de Empresas) de la Universidad Estatal de Milagro no registran aceptables niveles de autoestima y autoeficacia, afectando al proceso de generación y fortalecimiento de habilidades gerenciales".

Cuando se asimilan los procesos de comunicación en las aulas universitarias fortalecida con la comunicación no verbal, podemos observar a través de ella como determinados estudiantes van desarrollando un nivel de autoestima que les permite liberarse de esas cadenas que luego le aportan al momento de desarrollar su autoeficacia que traen consigo. Peter F. Drucker menciona respecto de los ejecutivos eficaces "lo único que tienen en común es la habilidad de conseguir que se hagan las cosas" (Drucker, 2012, 30), en esta línea se aspira aportar en la Unidad Académica a formar administradores eficaces, para lo cual se debe trabajar en las limitaciones que tienen los estudiantes, para liberarlos de estas; resultando imprescindible lograr un primer paso y es la conciencia de la existencia de estas limitaciones por parte de ellos, lo cual generaría la búsqueda potencial de soluciones.

En muchas oportunidades se ha conversado con los estudiantes para identificar las causas potenciales de sus comportamientos 
en clases, caracterizados por limitadas participaciones proactivas y en algunos casos con una marcada inseguridad, mencionan que son varias estas causas y que una de ellas y de mayor impacto es no haber generado en la secundaria el hábito de la lectura, el mismo que estiman se lo podría haber logrado, no sólo asignando tareas de lectura sino identificando textos que agreguen valor o que los motiven a la misma, sugieren la aplicación de la metodología de los talleres de lectura.

Todas las diferentes opciones que implican la propuesta académica son importantes, porque se asume que es producto de un proceso de investigación, de análisis y debate, en esta oportunidad se aporta con el presente trabajo respecto del comportamiento de los estudiantes que ingresan a la Unidad Académica de Ciencias Administrativas. Unidad en donde se forman a los futuros administradores de las empresas o Jefes-líderes que optimizarán la gestión de sus equipos, de su talento humano, aportando de esta manera a la eficacia de estos entes, cuyo éxito brindará el bienestar aspirado que aporte a una sociedad.

En esta línea Robert Sutton en su libro Buen Jefe Mal Jefe menciona que "El éxito o el fracaso de cada jefe depende de lo bien - lo mal que gestionen las relaciones humanas" (Sutton, 2011, 19), por este motivo nuestra preocupación en la existencia de niveles aceptables de motivación que deben registrar los estudiantes en la Universidad.

Como se menciona, son varias las causas que limitan el rendimiento de los estudiantes de la Unidad Académica de Ciencias Administrativas de la Universidad Estatal de Milagro al iniciar su proceso universitario, esto genera inclusive resistencias al cambio. Para efectos de la presente investigación nos concentraremos en la importancia de la asimilación de textos y la calidad de los mismos tanto en el segundo como en tercer nivel de educación. Lo expuesto anteriormente se vincula o enmarca con lo mencionado por Peter Drucker, quien afirmó “ El equilibrio entre el cambio y la continuidad exige una labor constante en materia de innovación" (Drucker, 1999, 129).

En base a los planteamientos anteriores, los autores tomaron la decisión de escribir un artículo vinculado con determinadas experiencias que han asimilado en su gestión como docentes y cuyos resultados observamos que cada semestre tienden a repetirse.

Un libro para un estudiante en la Universidad se debe constituir en una oportunidad para crecer personal y profesionalmente, los profesores aportan a este objetivo cuando estratégicamente los incluyen en los programas de estudio. En este sentido cuando formamos parte del equipo que trabajamos en los tres primeros semestres con los estudiantes de Administración de Empresas, debemos aportar al fortalecimiento de las habilidades blandas que caracterizan a los empleados considerados "Estrellas", como son: La Conciencia de sí mismo, Autorregulación, Motivación, empatía y Habilidades sociales (Goleman, 2004, 33), seleccionando textos que fortalezcan su proceso motivacional, fortaleciendo su carácter, aportando eficazmente a lo que Robbins identifica como habilidades técnicas, humanas y conceptuales (Robbins, 2009, 8).

Precisamente en la etapa inicial de los programas de tercer nivel se aprecia como inciden en el comportamiento humano de los integrantes de la Unidad Académica de Ciencias Administrativas de la sección nocturna, libros como: "Aprendiendo a Quererse a sí mismo", autor Walter Riso (Riso, 2003), "El factor Fred", Autor Mark Sanborn (Sanborn, 2008) y "Los Siete Hábitos de la Gente altamente Efectiva" de Stephen Covey (Covey, 2009). 
Con estos antecedentes se plantea las siguientes preguntas de investigación, con el propósito de obtener información que nos permita confirmar las mismas o reorientar la investigación.

- ¿La eficaz selección y lectura de textos en la secundaria permitirán que determinados estudiantes de la Unidad Académica de Ciencias Administrativas (Administración de Empresas) de la Universidad Estatal de Milagro reflejen óptimos niveles de autoestima y autoeficacia?

- ¿De qué manera la lectura en la secundaria de textos motivacionales aporta al desarrollo personal del estudiante y los prepara para un proceso universitario futuro?

- ¿Cómo influye la lectura de los libros Aprendiendo a quererse a sí mismo de Walter Riso, Los Siete Hábitos de la Gente Altamente Efectiva de Stephen Covey y el libro El Factor Fred de Mark Sanborn durante los primeros tres primeros semestres del proceso universitario en La Unidad Académica de Ciencias Administrativas (Administración de Empresas) de la Universidad Estatal de Milagro en los niveles autoestima, autoconcepto y autoeficacia de los estudiantes?

\section{Metodologia}

Las características principales de la presente investigación son: no experimental, exploratoria y descriptiva. No experimental porque no existe manipulación deliberada de las variables en estudio, solo se observan los fenómenos en su ambiente natural que es el aula, para después analizarlos. Exploratoria porque muy poco se sabe sobre la materia al principio del proyecto. Descriptiva porque se basa en la descripción del fenómeno a partir de la obtención de los datos.

La investigación enfocada de esta manera, facilitó explicar y caracterizar la realidad de la gestión del aprendizaje del estudiante y su relación con el clima de aula en el cual se desarrolla el proceso educativo, de tal manera, que hizo posible conocer el problema de estudio tal cual se presenta en la realidad, para que los docentes tomen en cuenta a partir de esta investigación. La investigación es también inductiva, ya que la inducción es el razonamiento, a partir de uno o varios juicios particulares para obtener una conclusión de aplicación general.

También se realizó búsqueda de información, antes, durante y posteriormente a la aplicación de los instrumentos de recolección de la información Este marco teórico-metodológico, permitió analizar, interpretar y discutir los resultados. Finalmente, se redactaron las conclusiones y recomendaciones.

A efectos de estructurar la investigación que permita responder las preguntas planteadas, se elaboró un cuestionario de preguntas que serán aplicadas a la población que está integrada por un total de 110 estudiantes que comprenden a los alumnos de la Unidad Académica de Ciencias Administrativas (Administración de Empresas) desde el tercer al séptimo nivel, no se considera los dos primeros semestres porque no cuentan o registran conocimiento parcial respecto del proceso de asimilación de los textos que son motivo de esta investigación y no se incluyó el octavo semestre ya que por procesos administrativos en el periodo octubre 2014- febrero 2015 no se registra este nivel. Paralelamente se entrevistó a un grupo de profesionales egresados de esta Unidad Académica que se encuentran ejerciendo su profesión de una manera destacada, quienes fueron entrevistados con el propósito de contrastar sus opiniones con los resultados de las encuestas anteriormente comentadas y de esta manera fortalecer las conclusiones respecto a los resultados encontrados. 


\section{Análisis de Resultados}

A continuación se analizan los resultados de la investigación.

En cuanto a la opinión de los estudiantes con respecto a cómo influye una selección eficaz de textos de lectura en la secundaria, la mayoría (99\%) opina que si en la secundaria se incentiva en mayor grado la lectura de libros, esto aportaría en el desarrollo de la autoestima y la autoeficacia del estudiante universitario. Ver Figura 1.

\section{Figura \\ Influencia de la eficaz selección y lectura de textos en la secundaria en su etapa universitaria.}

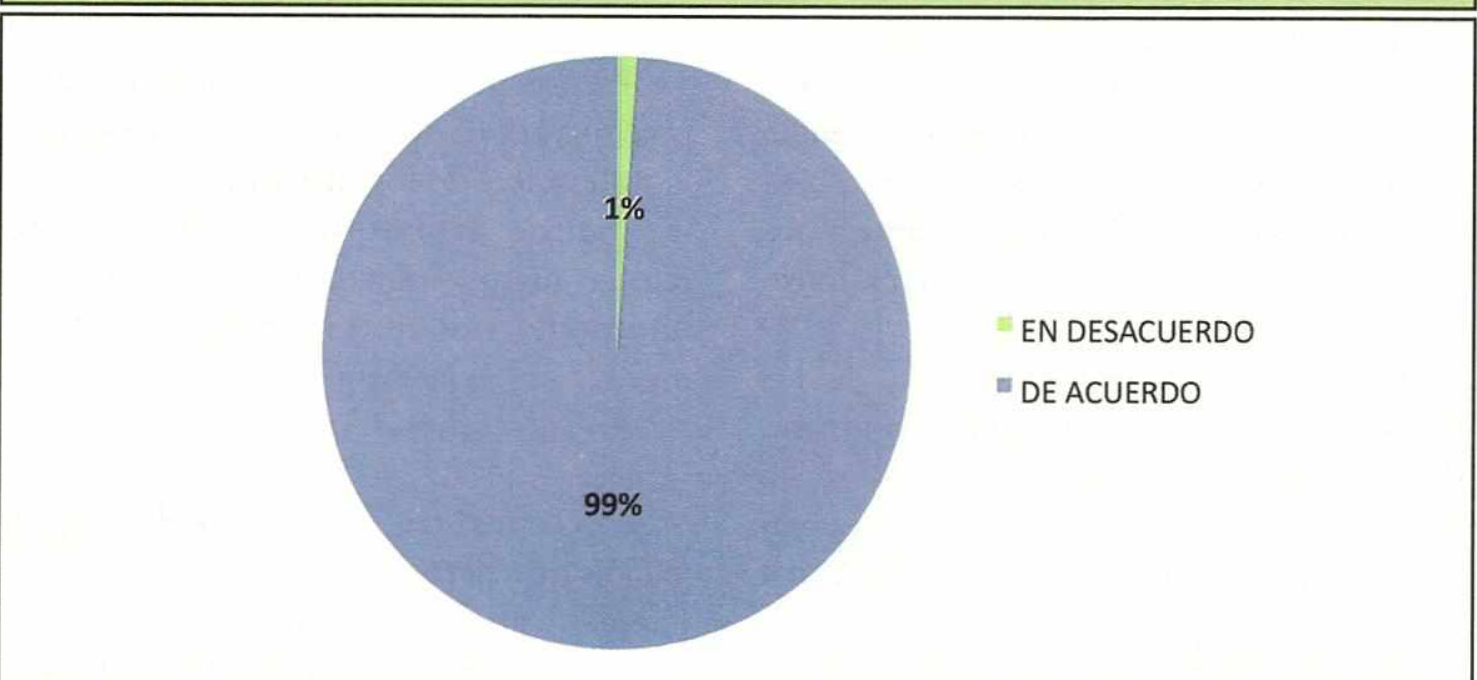

Fuente: Encuesta en la Universidad Estatal de Milagro

En varias oportunidades los docentes observan cómo el hábito de la lectura no se constituye en una fortaleza de determinados estudiantes y cómo esto incide en sus resultados, esta situación se la asimila como una consecuencia y no como una causa, por este motivo se consultó a quienes hoy se encuentran en la Universidad si se aportaría al desarrollo de la autoestima y la autoeficacia del estudiante universitario, si en la secundaria se incentivaba en mayor grado la lectura de libros. Los resultados son contundentes, el $99 \%$ de las personas consultadas opina estar de acuerdo que en la secundaria se debe propender en mayor grado a la generación del hábito de la lectura, para generar una mayor seguridad y oportunidades para quienes asimilan textos estratégicamente seleccionados, no se trata de un tema de cantidad, es un tema de calidad.

Queda latente la opinión de John C.
Maxwel, quien menciona que "La persona que capacita imparte el poder" (Maxwel, 2012, 75), lo que en nuestra opinión implica que la necesidad del establecimiento de un plan estratégico que no sólo establezca los textos, sino que los profesores reciban el entrenamiento respectivo que logre un mejor rendimiento de la capacitación.

Para estudiar lo relacionado con la lectura en la secundaria de textos motivacionales y su influencia en el desarrollo personal del estudiante y en la preparación para un proceso universitario futuro, los estudiantes fueron consultados en relación a tres textos con los cuales se trabaja en la Unidad Académica de Ciencias Administrativas vinculados con procesos motivacionales y de desarrollo profesional como son: "Aprendiendo a quererse a sí mismo", "Factor Fred" y "Los 7 hábitos de la gente altamente efectiva”, en la Figura 2 se presentan los resultados. 


\section{Figura 2}

\section{Libros que deben ser estudiados a nivel de secundaria.}

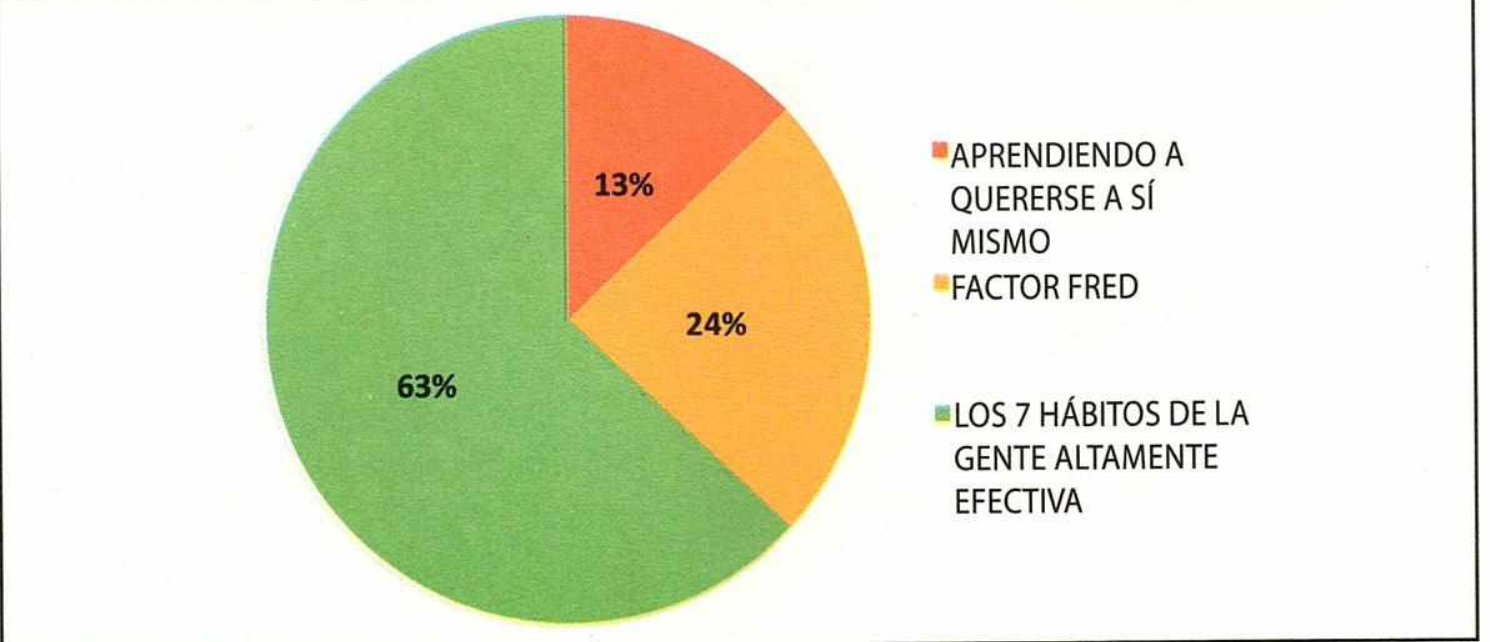

Fuente: Encuesta en la Universidad Estatal de Milagro

En Ingeniería Comercial durante los primeros semestres se asimilan los tres textos antes comentados y que se constituyen en las alternativas que se ofrecieron como respuestas en esta pregunta, para que los estudiantes elijan basados en su experiencia, ya que ellos los han asimilado previamente y que estiman agregaría valor si alguno de estos libros se los hubiera conocido en la secundaria previamente. Cada texto tiene sus características que influyen en las percepciones que tienen los estudiantes, sobre la motivación nos permitió determinar que el $71 \%$ estima que el libro cuyo conocimiento previo aportaría o los prepararía de mejor manera para el proceso universitario seria "Aprendiendo a Quererse a sí mismo”, en segunda posición nos encontramos con la alternativa del libro "El Factor Fred" con un 15\% y como tercera opción "Los Siete Hábitos de la Gente Altamente Efectiva”. Los autores están de acuerdo con los resultados obtenidos, como se ve posteriormente, existe una especie de secuencia lógica que vincula inicialmente con el orden establecido por los estudiantes encuestados, esto es "De adentro hacia afuera" como lo diría Stpehen Covey (Covey, 2009, 23).

Asimilar el libro Aprendiendo a quererse a sí mismo" fortalece la generación del lide- razgo, Warren Bennis comenta "Para convertirse en líder, uno necesita convertirse en uno mismo, ser el creador de su propia vida" (Bennis, 1990, 41). De igual manera se reflexiona sobre la necesidad de preocuparnos en nosotros mismos y esto incide en las fortalezas que deben tener los potenciales administradores, Michael Niehaus/Roger Wisniewski comentan "Esta preocupación por sí mismos, incluso hoy, es la base de cualquier acción empresarial, el que no es capaz de asumir responsabilidad por sí mismo, tampoco lo puede hacer por otras personas" (Niehaus, 2012, 39).

Como se menciona en el libro "Focus" (Goleman, 2013, 111): "El alto nivel de autocontrol no solo predice mejores calificaciones sino también un buen equilibrio emocional, mejores destrezas interpersonales, sentido de seguridad y adaptabilidad".

Para fortalecer esta investigación o validar los resultados obtenidos en el aspecto anterior, se agregó otro aspecto relacionado con la selección del libro que debería tratarse sólo en el proceso universitario. Ver Figura 3. 


\section{Figura 3}

\section{Libro que debe tratarse en el proceso universitario}

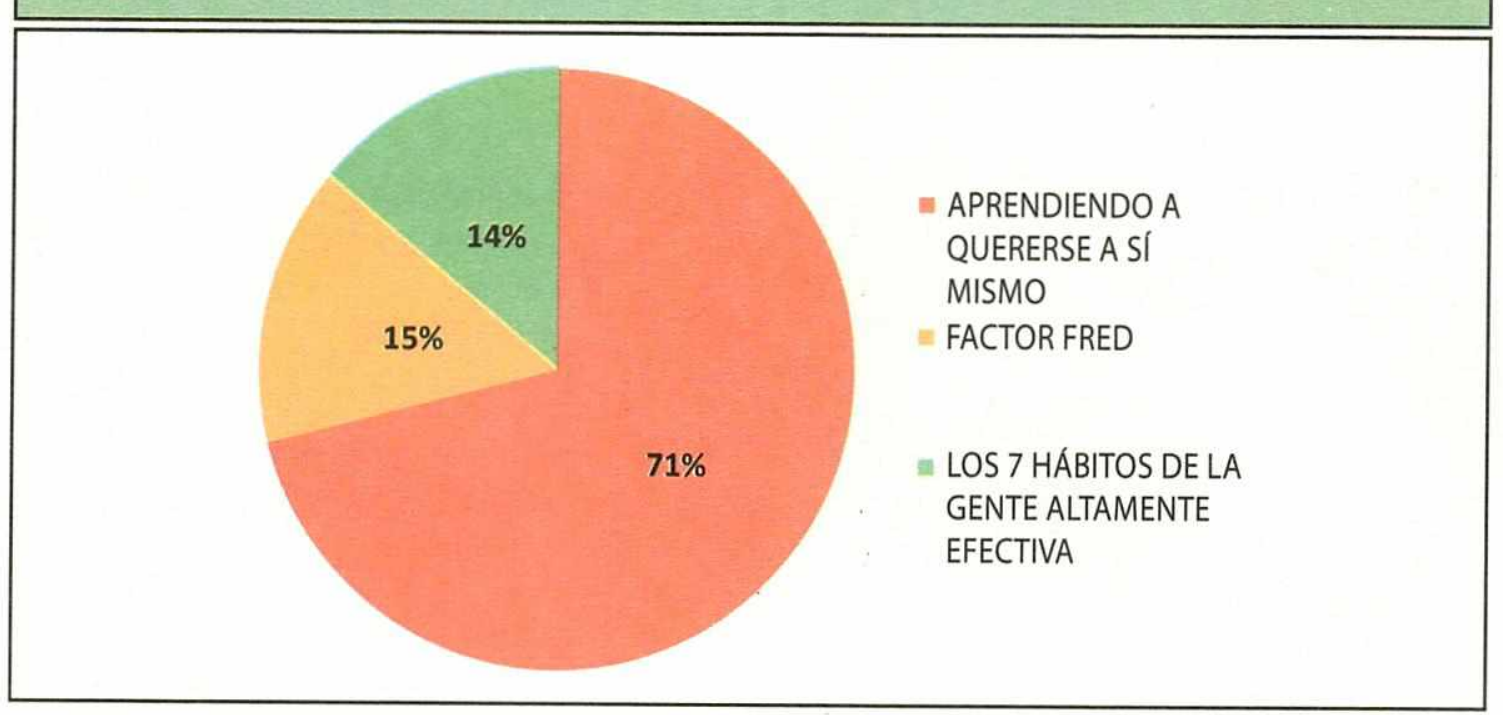

Fuente: Encuesta en la Universidad Estatal de Milagro

Cuando se analizan las respuestas conseguidas, se determina que esta pregunta genera un lógico inverso resultado al obtenido en la pregunta presentada anteriormente, lo cual no sólo permite conocer nuevos resultados, sino validar los obtenidos previamente. Los estudiantes estiman que en primer lugar con $63 \%$ o 70 personas, se lo debe considerar al libro "Los Siete Hábitos de la Gente altamente efectiva” en segundo lugar con un $24 \%$ o 26 personas al libro "El Factor Fred". Efectivamente, estos dos libros como se presenta posteriormente, si bien fortalecen la motivación, es indudable que agregan valor al perfil del Administrador de empresas en su formación. Y sólo un $13 \%$ o 14 personas estiman que el libro "Aprendiendo a quererse a sí mismo" seria la opción conveniente.

Se estima que Covey en su obra sugiere construir un liderazgo basado en las relaciones interpersonales, sus hábitos como los de: Sinergia, Procure Comprender y Ganar - Ganar, se alinean a este sentido, Marcus Buckinhan y Curt Coffman, comparten esta visión al mencionar " Las actividades más esenciales humanas son: percibir, juzgar, crear y construir relaciones" (Buckingham, 2012, 27). La obra de Covey permite que los futuros administradores trabajen desde su interior teniendo en consideración el logro de la interdependencia, obteniendo el respeto del personal a cargo y no el temor, Xavier Guix expresa "El cargo no lo es todo. La autoridad no depende de lo que pone en una tarjeta de visita o placa o en la puerta de despacho. La autoridad se desprende de una presencia que inspira, de la sensibilidad en el trato con los demás” (Guix, 2013, 21).

Los datos obtenidos no sólo permiten validar la hipótesis, adicionalmente brindan ya una opción clara de un texto que se estima pertinente asimilarlo en la secundaria.

Aporta en el análisis de estos resultados alcanzados si paralela y sinérgicamente se realizan entrevistas a profesionales egresados de la Unidad Académica de Ciencias Administrativa de la UNEMI, quienes se encuentran laborando en el sector financiero, al ser consultados:

¿Le habría aportado en su proceso motivacional y en su proyección universitaria el haber tenido la oportunidad de haber asimilado previamente la obra de Walter Riso?, mencionaron lo siguiente:

Ing. Ivannia Ormeño: "La etapa secundaria está marcado en la mayor parte por la 
incertidumbre si no estamos correctamente guiados, se debería en esta etapa no solo prepararnos en la parte académica, sino también en la personal a través de la lectura de libros como este, trabajar en este último concepto desde inicios de la misma e irla fortaleciendo a medida que vamos avanzado, para que al momento de proyectarnos una carrera universitaria lo hagamos consciente de nuestras capacidades y habilidades, de conocernos y saber el valor imprescindible de la autoestima".

Ing. Carlos Murillo: "Realmente creo que me habría aportado mucho en el aspecto motivacional para así haber podido enfrentar de mejor manera situaciones que se presentarían en el futuro. $Y$ aunque en la etapa de secundaria hubiese sido un gran aporte lo sigue siendo para cada día, ya que debemos reinventarnos para afrontar todo tipo de circunstancias que nos rodean y para esto debemos estar seguros de: ¿Quiénes somos, qué es lo que podemos lograr, qué es lo que debemos mejorar, qué es lo que debemos potenciar más en nosotros?".

La opinión de los egresados coincide con el planteamiento de Allan Percy quien menciona "Amarse a sí mismo es el comienzo de un idilio que durará toda la vida" (Percy, 2011, 76), definitivamente la secundaria se constituye en una oportunidad que se debe aprovechar al máximo cuando de motivación hablamos, precisamente en este sentido no se puede quedar impávido ante una necesidad latente, continua.

A continuación se presentan los resultados de cómo influye en los niveles de autoestima, autoconcepto y autoeficacia de los estudiantes de la Unidad Académica de Ciencias Administrativas (Administración de Empresas) de la Universidad Estatal de Milagro la lectura de los libros Aprendiendo a quererse a sí mismo de Walter Riso, Los Siete Hábitos de la Gente Altamente Efectiva de Stephen Covey y el libro El Factor Fred de Mark Sanborn durante los primeros tres primeros semestres del proceso universitario.

A efectos de optimizar la investigación se consultó a los estudiantes respecto del aporte que tuvieron en ellos la lectura de los libros en su programa de estudios, segregando en tres preguntas vinculadas entre sí, la primera con la Autoestima, la segunda con el Autoconcepto y Autoeficacia y la tercera de manera general con su Desarrollo Personal y Profesional. En las Tablas 1, 2 y 3, y en las Figuras 4,5 y 6 se pueden observar los resultados obtenidos debidamente contrastados con las opiniones de egresados de la Unidad Académica.

En cuanto al nivel de aporte a la Autoestima, los mejores resultados lo obtiene el libro Aprendiendo a quererse a sí mismo los estudiantes estiman que aportó a su desarrollo en Alto nivel en el $87 \%$, y $11 \%$ en mediano nivel, con lo cual si analizamos de manera general y sumamos ambas respuestas se registraría un global positivo del $99 \%$. En segunda y tercera posición con un empate del $80 \%$ nos encontramos con los textos de Covey y Sanborn, los encuestados opinan que aportaron en alto nivel a su desarrollo, diferenciándose en sus resultados cuando se conoce que el $18 \%$ consideran haber recibido un aporte en mediano nivel cuando efectuaron la lectura del libro Los siete hábitos de la gente altamente efectiva y $16 \%$ los que se identifican con el libro el Factor Fred.

Rosa Guamán, egresada de la Unidad Académica de Ciencias Administrativas menciona respecto del aporte que en ella tuvo la obra de Riso: "Partiendo del hecho de comprender que la vida no hay que vivirla pensando en función de los demás con el fin de "caerle bien a todo el mundo" y que no hay mayor motivación para el desarrollo tanto intelectual como físico de un ser humano que el sentirse autosuficiente como tal, por lo general las personas nos imponemos un punto de control que nos frena y hace que evitamos hacer cosas por temor a equivo- 
carnos, tenemos derecho a autovalorarnos y elegir lo mejor para nuestra propia realización personal y profesional, en este sentido el libro apoya directamente."

Otra Egresada como Diana Guin, menciona: El Factor Fred en mí aportó mucho, tuve la oportunidad de leerlo en segundo semestre en la universidad y desde ese día se quedó grabado en mi memoria. Es un libro que trae muchas enseñanzas y ejemplos positivos de cómo debe ser nuestro actuar en diferentes ámbitos de la vida, Fred me enseñó principalmente que cada labor que realice debe crear un valor continuo, que el objetivo está en marcar la diferencia y ser ejemplo para los demás."

La participación de las egresadas permitió entender la opinión de John Maxwel en su libro "Lider de 360 grados" cuando analiza lo que él considera como los Mitos de dirigir una organización desde la zona intermedia, en este sentido comenta que hay personas en las organizaciones que no crecen en las mismas, no participan activamente ya que estiman que no es el momento oportuno, porque existe la opinión errada de que "No se puede dirigir si no estoy en la cima" (Maxwel, 2007, 4).

\begin{tabular}{|c|c|c|c|c|c|c|c|c|}
\hline \multicolumn{9}{|c|}{$\begin{array}{l}\text { Tabla \# } 1 \\
\text { Aporte a la Autoestima. }\end{array}$} \\
\hline \multirow{3}{*}{ LIBROS } & \multicolumn{8}{|c|}{ Grado de aporte en el desarrollo de la autoestima } \\
\hline & \multicolumn{2}{|c|}{ Alto nivel } & \multicolumn{2}{|c|}{ Mediano nivel } & \multicolumn{2}{|c|}{ Bajo nivel } & \multicolumn{2}{|c|}{ Nada } \\
\hline & Número & $\%$ & Número & $\%$ & Número & $\%$ & Número & $\%$ \\
\hline $\begin{array}{l}\text { Aprendiendo a } \\
\text { quererse a sí mismo }\end{array}$ & 96 & $87 \%$ & 12 & $11 \%$ & 1 & $1 \%$ & 1 & $1 \%$ \\
\hline $\begin{array}{l}\text { Los } 7 \text { hábitos de la } \\
\text { gente altamente } \\
\text { efectiva }\end{array}$ & 88 & $80 \%$ & 20 & $18 \%$ & 0 & $0 \%$ & 2 & $2 \%$ \\
\hline Factor fred & 88 & $80 \%$ & 18 & $16 \%$ & 1 & $1 \%$ & 3 & $3 \%$ \\
\hline
\end{tabular}


Figura 4

Nivel de aporte a la Autoestima.

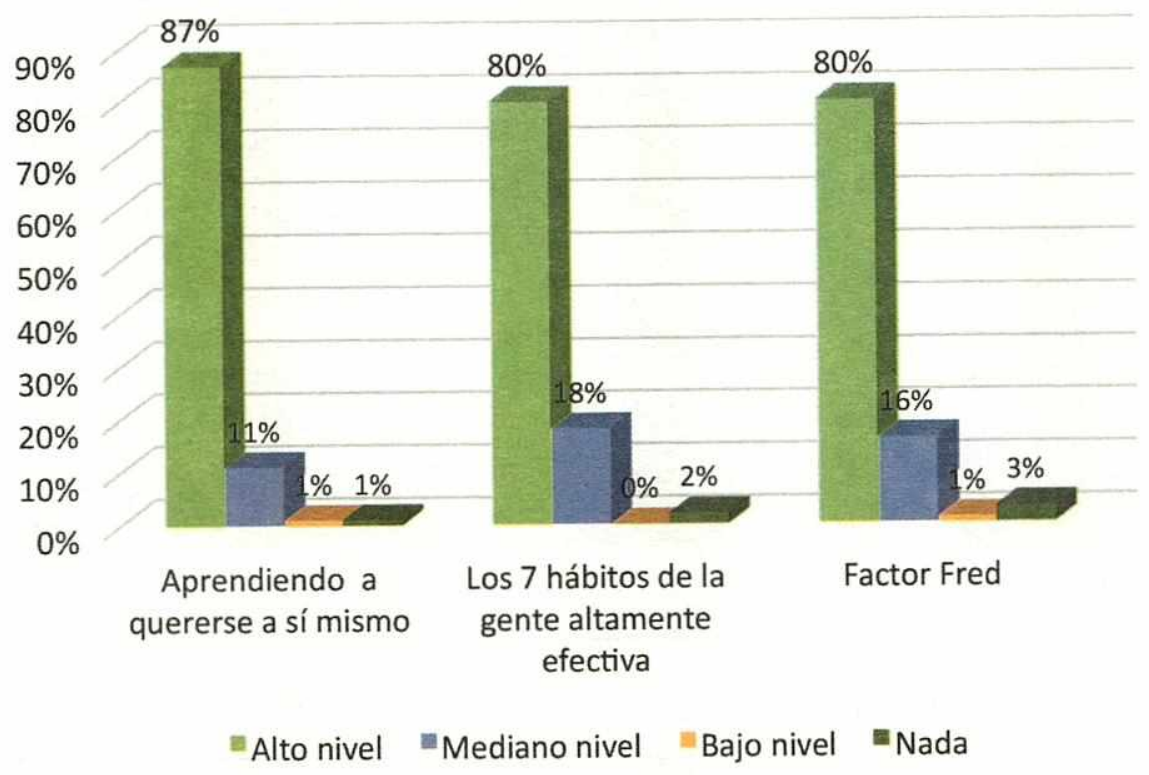

Fuente: Encuesta en la Universidad Estatal de Milagro.

Con respecto al nivel de aporte al Autoconcepto y la Autoeficacia, las personas encuestadas le brindan una respuesta positiva del $100 \%$ a la obra de Riso ya que el $78 \%$ estiman haber recibido un aporte en alto grado a su desarrollo y el $21 \%$ en mediano nivel, los textos de Covey. En segunda posición se registra la obra de Covey con un
$98 \%$ distribuido en un $79 \%$ con un efecto en alto nivel a su desarrollo y la diferencia lo considera en un mediano nivel. Por último el Factor Fred con un 97\% recibe una respuesta favorable, integrada mayoritariamente esta tendencia con un $83 \%$ que califican el aporte en un nivel superior a su desarrollo.

Tabla \# 2

Aporte al autoconcepto y la autoeficacia.

\begin{tabular}{|c|c|c|c|c|c|c|c|c|}
\hline \multirow[t]{3}{*}{ LIBROS } & \multicolumn{8}{|c|}{ Grado de aporte en el desarrollo del autoconcepto y la autoeficacia } \\
\hline & \multicolumn{2}{|c|}{ Alto nivel } & \multicolumn{2}{|c|}{ Mediano nivel } & \multicolumn{2}{|c|}{ Bajo nivel } & \multicolumn{2}{|c|}{ Nada } \\
\hline & Número & $\%$ & Número & $\%$ & Número & $\%$ & Número & $\%$ \\
\hline $\begin{array}{l}\text { Aprendiendo a } \\
\text { quererse a sí mismo }\end{array}$ & 86 & $78 \%$ & 23 & $21 \%$ & 1 & $1 \%$ & 0 & $0 \%$ \\
\hline $\begin{array}{l}\text { Los } 7 \text { hábitos de la } \\
\text { gente altamente } \\
\text { efectiva }\end{array}$ & 87 & $79 \%$ & 20 & $18 \%$ & 1 & $1 \%$ & 2 & $2 \%$ \\
\hline Factor fred & 91 & $83 \%$ & 14 & $13 \%$ & 1 & $1 \%$ & 4 & $4 \%$ \\
\hline
\end{tabular}

Fuente: Encuesta en la Universidad Estatal de Milagro.

Elaborado por autor. 
Figura 5

Nivel de aporte al autoconcepto y la autoeficacia

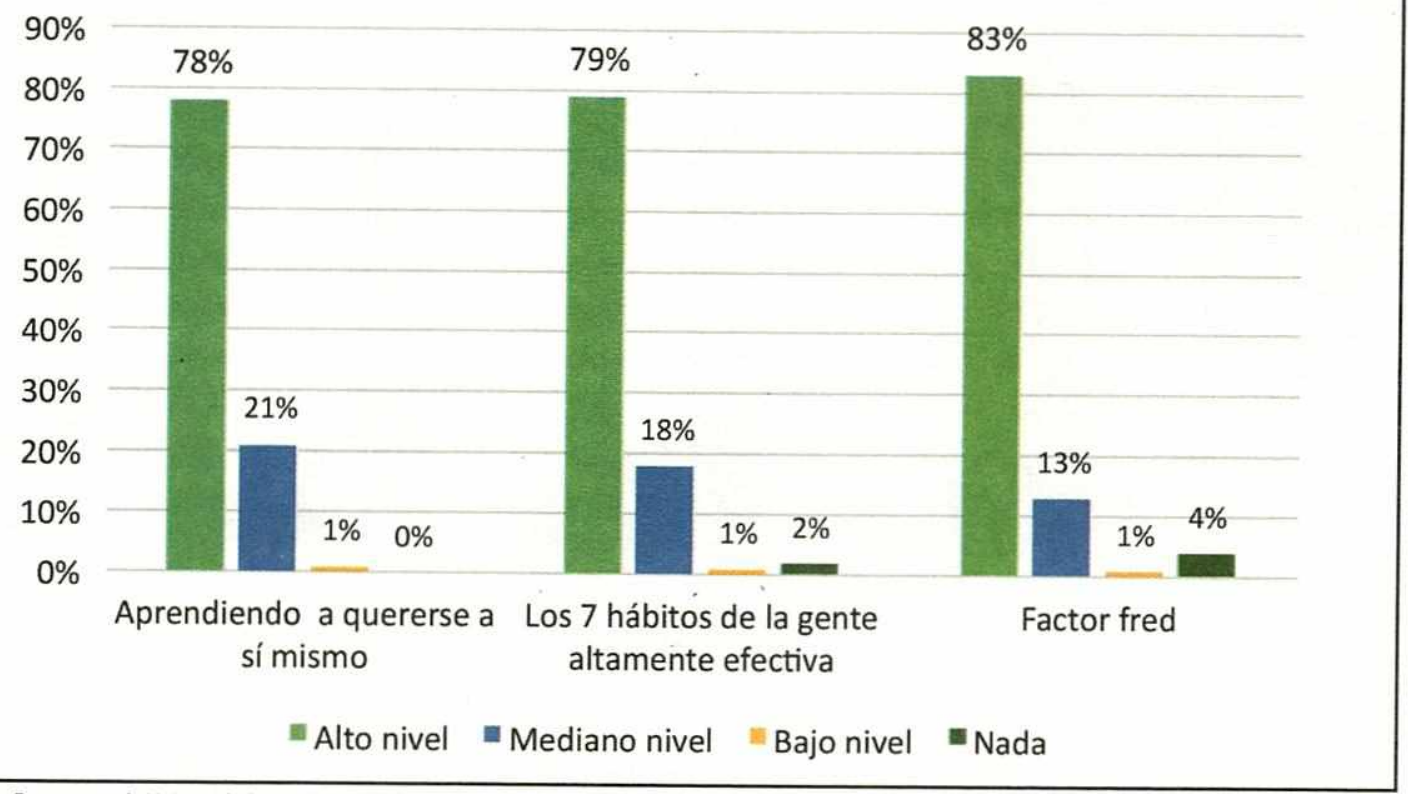

Fuente: Encuesta en la Universidad Estatal de Milagro.

En cuanto al aporte al Desarrollo Personal y Profesional, con el propósito de "hilar fino" respecto del término "Desarrollo" se incluyó una pregunta que buscaba conocer de los encuestados sus percepciones respecto del impacto que generan en ellos los libros analizados, marcando una diferencia o puntualizando una diferencia entre el Desarrollo Personal y el Desarrollo Profesional, asimilando el personal con los procesos motivacionales, de autoestima y autoconcepto y el Profesional con los de la Autoeficacia.

Los resultados obtenidos permiten conocer que la posición mayoritaria es percibir un efecto global respecto al Desarrollo, esto es, se aporta de manera conjunta a los dos desarrollos, el personal y el profesional ya que el $74 \%$ estiman que el Factor Fred genera este doble aporte, seguido por la obra de Walter Riso con un $68 \%$ y estrechamente cercano encontramos con un $67 \%$ al libro de Sanborn. es necesario de igual manera conocer o analizar la opinión de quienes consideran que existe un solo aporte y no uno general, esto se puede observar en quienes consideran los libros de los siete hábitos de la gente altamente efectiva y el Factor Fred con una influencia más profesional que personal con 17 y $11 \%$ respectivamente y Riso se identifica exclusivamente con el desarrollo personal o motivacional en un $25 \%$.

Los estudiante asimilan el "El Factor Fred" y aprecian el potencial que brinda su mensaje, marcando la diferencia y agregando valor en lo que hacen, constituyéndose inclusive en nutrientes al momento de emprender, de liderar, Mario Enrique Uribe menciona: "El sector educativo es fundamental en toda transformación cultural. Se trata de desarrollar el potencial emprendedor de cada individuo, acompañándolo en el proceso de descubrimiento personal" (Uribe, 2013, 26). Se considera que la obra de Samborn aporta en la generación de creatividad e innovación o lo que Edward de Bono lo identifica como "Pensamiento Lateral", que permite ver de una manera distinta una realidad (De Bono, 2008, 25).

Cuando alguien se convierte en un "FRED", marca la diferencia en sus rela- 
ciones interpersonales, asimila una visión de servicio, pensando en el prójimo, generando en ellos una reflexión respecto de su comportamiento parar ser mejores, Annie McKee, Richard Boyatzis y Frances Johnston al respecto mencionan "la capaci- dad para movilizar la energía a fin de mejorar la calidad de vida de la gente es una de las aptitudes más fascinantes y cruciales para entender y, en último extremo, dominar" (Boyatzis, 2008, 11).

\section{Thabla \# 3}

Aporte al Desarrollo Personal y Profesional.

\begin{tabular}{|c|c|c|c|c|c|c|c|c|}
\hline \multirow{3}{*}{ LIBROS } & \multicolumn{8}{|c|}{ Grado de aporte en cuanto a: } \\
\hline & \multicolumn{2}{|c|}{$\begin{array}{c}\text { Su desarrollo } \\
\text { personal }\end{array}$} & \multicolumn{2}{|c|}{$\begin{array}{c}\text { Su desarrollo } \\
\text { profesional }\end{array}$} & \multicolumn{2}{|c|}{$\begin{array}{c}\text { Su desarrollo } \\
\text { personal y } \\
\text { profesional }\end{array}$} & \multicolumn{2}{|c|}{$\begin{array}{c}\text { No aportó a su } \\
\text { desarrollo personal } \\
\text { y profesional. }\end{array}$} \\
\hline & Número & $\%$ & Número & $\%$ & Número & $\%$ & Número & $\%$ \\
\hline $\begin{array}{l}\text { Aprendiendo a } \\
\text { quererse a sí mismo }\end{array}$ & 28 & $25 \%$ & 4 & $4 \%$ & 75 & $68 \%$ & 3 & $3 \%$ \\
\hline $\begin{array}{l}\text { Los } 7 \text { hábitos de la } \\
\text { gente altamente } \\
\text { efectiva }\end{array}$ & 13 & $12 \%$ & 19 & $17 \%$ & 74 & $67 \%$ & 4 & $4 \%$ \\
\hline Factor Fred & 15 & $14 \%$ & 12 & $11 \%$ & 81 & $74 \%$ & 2 & $2 \%$ \\
\hline
\end{tabular}

Fuente: Encuesta en la Universidad Estatal de Milagro.

Elaborado por autor.

\section{Figura 6}

Nivel de aporte al Desarrollo Personal y Profesional.

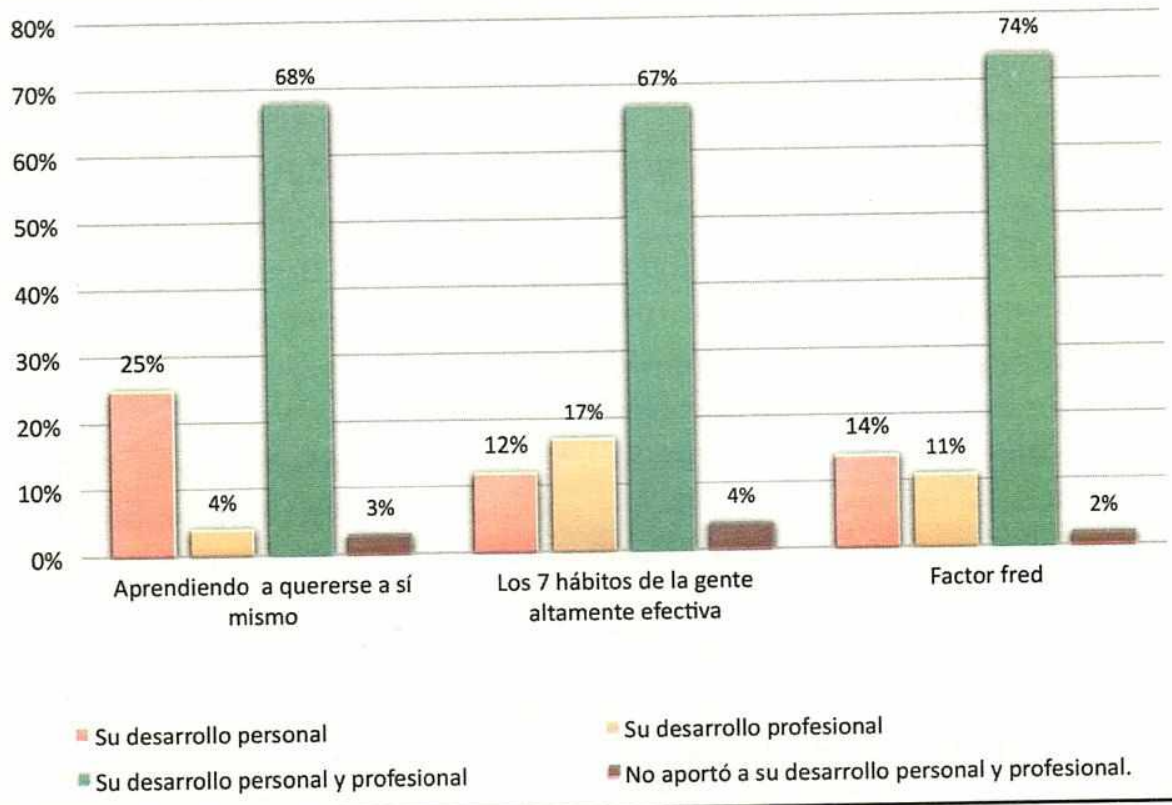


Las opiniones de los egresados agregan valor a los datos obtenidos y generan sinergia cuando se conoce que:

Carlos Murillo: Respecto del Factor Fred menciona: "El hombre puede convertir el mundo ordinario en que vive en algo extraordinario si empieza por sí mismo, el libro si aportó mucho en mi desarrollo personal y profesional. Algo muy importante que señala el libro es que debemos reinventarnos a nosotros mismos con regularidad, es decir superar lo que ya habíamos hecho o logrado".

Respecto de Covey la Ing. Ormeño comenta: "Lo más importante de auto educarnos es que en un libro podemos llegar a encontrar no solo una superación profesional sino también personal, especialmente en los siete hábitos de Covey, cuando lo leí por primera vez todos los ejemplos me llevaban a la vida diaria ya que no tenía mayor experiencia laboral y es ahí donde comienza el cambio en tu entorno y la forma de actuar, hoy que ya cuento con la segunda, comprendo que muchas veces escuchaba para responder y no para comprender"

Ram Charan identifica lo que según opinión es el Know How en su libro de similar nombre, como la "pericia" (Charan, $2009,1)$ que debe contar todo administrador, precisamente en la opinión de los egresados, se percibe este Know How de manera implícita, estimando en su generación tiene mucha incidencia la asimilación de los textos que han sido motivo de este trabajo de investigación y la respectiva aplicación de esta información en su diario vivir.

\section{Conclusiones}

Una vez identificado el problema y planteada la hipótesis haciendo acopio de la experiencia de los autores se plantearon las preguntas de investigación que se constituyeron en los nutrientes de las encuestas cuyos resultados permitieron validar estos planteamientos y que se los resume a continuación:

- Resulta necesario fortalecer en la secundaria el hábito de la lectura, para lo cual la selección de textos que agreguen valor fortalecen este objetivo, estimándose que se agrega valor cuando los profesores utilizan técnicas como: los talleres de lecturas de textos estratégicamente seleccionados, para que el estudiante los asimile con expectativas de mejoras.

- En opinión de los autores uno de los principales objetivos de los profesores en la universidad, es lograr que sus estudiantes descubran sus talentos ocultos, fortaleciendo sus habilidades gerenciales, preocupa los comentarios de Marcus Buckinham y Donald. O. Clifton, respecto de determinadas personas que "no buscan activamente el conocimiento y las destrezas para fortalecer sus talentos", ya que es una realidad que debemos enfrentar y solucionar (Buckingham, 2013, 43), esta realidad es la zona confort.

- Es necesario propender a la lectura de textos motivacionales, si bien es cierto hemos analizado la situación de la Unidad Académica de Ciencias Administrativas de la Universidad Estatal de Milagro, la motivación es un elemento que está en todo ser humano, su fortalecimiento es necesario para mejorar el carácter de quienes siempre lo necesitaran.

- La extraordinaria obra del Autor Walter Riso "Aprendiendo a Quererse a sí mismo se la debe asimilar en la secundaria, esta conclusión es producto de la opinión de los estudiantes encuestados y los egresados entrevistados, a esto se suma la opinión de los autores del presente trabajo. 
- La Obra "Los Siete hábitos de la gente altamente efectiva" de Stphen Covey y "El Factor Fred" de Mark Sanborn, aportan al proceso motivacional e indiscutiblemente fortalecen el perfil profesional del estudiante de Administración de Empresas, estos textos se los deben asimilar durante los primeros tres semestres de la carrera.

- Un administrador debidamente fortalecido en sus habilidades blandas con la motivación necesaria para estructurar y guiar equipos de alto rendimiento aportará inclusive en lo que se conoce como la identificación de "Océanos Azules" (Mauborgne, 2012 , 4) o mercados no aprovechados, porque estará abierto a valorar los aportes de sus liderados.

- Cuando se trabaja en las habilidades humanas de los estudiantes se lograría inclusive una menor resistencia al cambio, evitando las zonas de confort, al respecto Daniel Goleman menciona "Los líderes visionarios son empáticos, tienen confianza en sí mismos y suelen actuar como agentes de cambio"(Goleman, 2005, 80).

\section{Recomendaciones}

Luego de haber culminado el presente trabajo de investigación se recomienda sustentado en las conclusiones obtenidas: que es necesario fortalecer el hábito de la lectura en la secundaria, para lo cual los docentes deben ser parte de un plan estratégico, que permita identificar las necesidades que se aspira satisfacer; en el proceso se deben seleccionar textos de acuerdo a estas necesidades y brindarles las respectivas capacitaciones a los docentes para vigorizar sus talentos, sobre todo, en la gestión de talleres de lecturas. Se recomienda que los textos "Aprendiendo a Quererse a sí mismos" se los asimile en la secundaria y que los textos "Los Siete hábitos de la gente altamente efectiva" y "El factor Fred" se los asimile en el tercer nivel o proceso universitario durante los primeros semes- tres. Si bien es cierto se analizó la situación de una Unidad Académica vinculada con la gestión comercial, se sugiere analizar la información existente que podría ser aplicada en otras por las características que esta implica.

Se debe estar preparado para aprovechar la oportunidad que brinda, la posibilidad de efectuar cambios en las percepciones de los estudiantes, varios de ellos presentan ideas erradas de lo que implica administrar y de lo que es liderar, como sabemos no es lo mismo pero si son complementarias, porque una administración sin liderazgo no generan eficacia, Chris Lowney menciona "Todos somos imperfectos: los demonios del egocentrismo, el ansia de poder, el temor o la falta de confianza acechan a todo líder. El liderazgo no consiste meramente en conocerse a sí mismo, sino en manifestar la voluntad de combatir sus demonios" (Lowney, 2015, 49).

\section{Bibliografia}

Bennis, W. (1990). ¿Cómo llegar a ser líder?, Grupo Editorial Norma, Bogotá Colombia.

Boyatzis, R.; Mackee, A. \& Johnston, F. (2008). Líder emocional. Ediciones Deusto, Barcelona - España.

Buckingham, M. \& Coffman, C. (2012). Primero, rompa todas las reglas. Editorial Buena Semilla, Bogotá - Colombia.

Buckingham, M.; Clifton, D. O. (2012). Ahora: descubra sus fortalezas. Editorial Buena Semilla, Bogotá - Colombia.

Covey, R. 2009. Los siete hábitos de la Gente Altamente. Efectiva. Paidos, México.

Charan, R. (2009). Know How. Grupo Editorial Norma, Bogotá - Colombia. 
De Bono, E. (2008). El pensamiento lateral práctico, Barcelona-España: Paidós Ibérica S.A.

Drucker, P. (1969.) El ejecutivo eficaz. Buenos Aires: Editorial Sudamericana S.A.

Drucker, P. (2002). Editorial Norma, Bogotá Colombia.

Fierro, L. (2015). Coaching Para Líderes, Ediciones Granica S.A., Buenos Aires Argentina.

Goleman, D. (2013). Focus. Ediciones B. Buenos Aires - Argentina.

Goleman, D. (2004). La Práctica de la Inteligencia Emocional. Editorial Kairos, Barcelona - España.

Goleman, D. \& Cherniss, C. (2005). Inteligencia emocional en el trabajo. Editorial Kairos, Barcelona - España.

Guix, X. (2013). Jefes. Editorial Conecta, Bogotá-Colombia.

Kahnweiler, J. (2010). El lider introvertido. Ediciones Urano S.A., Barcelona España.

Lowney, C. (2015). Papa Francisco. Ediciones Granica S.A., Buenos Aires - Argentina.

Mauborgne, R. \& Xhan Kim. (2012). La estrategia del océano azul. Grupo Editorial Norma, Bogotá - Colombia.

Maxwel, J. (2012). El ABC de la capacitación. Editorial V\&R, Buenos Aires - Argentina.

Maxwel. J. (2007). Líder de 360 grados. Editorial Lidere, Estados Unidos.
Muradep, L. (2015). Coaching para la transformación personal. Ediciones Granica S. A., Buenos Aires - Argentina.

Murnighan, K.J. (2013). Hacer: jNada!. Editorial Conecta, Cundinamarca - Colombia.

Niehaus, M. \& Wisniewski, R. (2012). El arte de preguntar: administracion por Sócrates, Panamericana Editorial, Bogotá - Colombia.

Percy, A. (2011). El Coaching de Oscar Wilde, Editora Gémenis, Bogotá- Colombia.

Ponti, F. \& Ferrás, X. (2008). Pasión por innovar. Grupo Editorial Norma, Bogotá Colombia.

Riso, W. 2003. Aprendiendo a quererse a sí mismo. Editorial Norma, Bogotá -Colombia.

Robbins, S. \& Judge, T. (2009). Comportamiento Organizacional. Décima tercera Edición. Editorial Pearson Education. Mexico.

Sanborn, M. (2008) El factor Fred. Editorial Norma, Bogotá - Colombia.

Sutton. R,I. (2011) Buen Jefe, Mal Jefe. Conecta, Barcelona- España.

Uribe, M. \& Reinoso, J. (2013). Emprendimiento y Empresarismo. Ediciones de la U, Bogotá - Colombia.

Zenger, J. \& Stinnett, K. (2013). El coach extraordinario. Profit Editorial, Barcelona - España. 


\section{Héctor Serrano Mantilla}

Ingeniero Comercial, MAE. Docente de tercer y cuarto nivel de la Universidad Estatal de Milagro UNEMI. Gerente de Negocios del Banco del Pacifico.

E-mail: hserranom@unemi.edu.ec

\section{Jesennia Cárdenas Cobo}

Docente en la carrera de Ingeniería en Sistemas Computacionales, Facultad Ciencias de la Ingenieria, Universidad Estatal de Milagro UNEMI.

E-mail: jcardenasc@unemi.edu.ec

\section{Mariuxi Vinueza Morales}

Máster en Administración y Dirección de Empresas. Docente en la carrera de Ingenieria en Sistemas Computacionales, Facultad Ciencias de la Ingenieria, Universidad Estatal de Milagro UNEMI.

E-mail: yovinueza@gmail.com

\section{Rodolfo Robles Salguero}

Máster en Administración de Empresas, Docente, Facultad Ciencias de la Ingenieria, Universidad Estatal de Milagro UNEMI. Director del Instituto de Postgrado de Universidad Estatal de Milagro UNEMI.

E-mail: rrobless@unemi.edu.ec 
